\title{
Effects of a single dose of ethanol on survival rate and angiogenesis of chick embryo
}

\author{
Zulifqar Ali Laghari", *, Ayaz Ali Samo1, *, Baradi Waryani²,Zameer Ali Palh ${ }^{2}$ *, Khalid Hussain \\ Lashari $^{2}$, Ghulam Murtaza Mastoi ${ }^{3}$, Gulshan Ara Sahato ${ }^{2}$, Tahira Jabeen Ursani ${ }^{4}$ \\ ${ }^{1}$ Department of Physiology, University of Sindh, Jamshoro, Sindh, Pakistan \\ ${ }^{2}$ Department of Fresh Water Biology and fisheries, University of Sindh, Jamshoro, Sindh, Pakistan \\ ${ }^{3}$ Centre for Environmental Sciences, University of Sindh, Jamshoro, Sindh, Pakistan \\ ${ }^{4}$ Department of Zoology, University of Sindh, Jamshoro, Sindh, Pakistan
}

Email address:

zameer_ali110@hotmail.com (Z. A. Palh),kh_lashari@yahoo.com (K. H. Lashari)

\section{To cite this article:}

Zulifqar Ali Laghari, Ayaz Ali Samo, Baradi Waryani,Zameer Ali Palh, Khalid Hussain Lashari, Ghulam Murtaza Mastoi, Gulshan Ara Sahato, Tahira Jabeen Ursani. Effects of a Single Dose of Ethanol on Survival Rate and Angiogenesis of Chick Embryo. Animal and Veterinary Sciences. Vol. 3, No. 1, 2015, pp. 8-11. doi: 10.11648/j.avs.20150301.12

\begin{abstract}
The developing Chick has been widely used as a model organism for research studies in developmental biology. Various aspects of ethanol (EtOH) toxicity during embryonic development have been documented in experimental studies. The studies on chick embryo address the effects of EtOH on craniofacial abnormalities and angiogenesis. The purpose of this study was to assess the effects of a single dose of ethanol on survival rate and angiogenesis of chick embryos during early stages of development. Chick eggs were incubated at $37^{\circ} \mathrm{C}$ in the humidified incubator, after 72 hours of incubation these eggs were removed from incubator, and treated with a single doses of 1\%,5\%,10\%, 13\% and 15\% EtOH. The effects on survival rate and angiogenesis were recorded on ethanol treated chick embryos. The survival rate was slightly reduced with $1 \%$ and $5 \%$ EtOH, however with increasing dose of $10 \%$ the survival rate was reduced to $64 \%$ and at $15 \%$ EtOH complete death was observed. Our results also indicate that treatment of EtOH with $1 \%$ and $5 \%$ did not have any obvious effects on vessels formation in comparison with the BSS treated embryos. However, treatment of chick embryo with 10\%, 13\% and 15\% EtOH severely inhibited the blood vessels formation.
\end{abstract}

Keywords: Chick Embryo, Angiogenesis, EtOH

\section{Introduction}

Ethanol (EtOH) is the main component of the alcohol. Alcohol is consumed frequently irrespective of gender. Some females consume alcohol even in pregnancy, which might result into Fetal Alcohol Syndrome (FAS) (Popova, Lange et al. 2011; Lange, Shield et al. 2013). Individuals suffering from FAS exhibit the spectrum of defects including Physical and Mental retardation, cognitive, behavioral and learning disabilities with identifiable facial dysmorphology (Popova, Lange et al. 2011; Lange, Shield et al. 2013). A number of studies report the use of animal models in the understanding of complexity of FAS and effects of EtOH on various developmental defects have been extensively studied on different animal models including mice (Nakatsuji and Johnson 1984), chick (Tufan, Abban et al. 2007), Xenopus (Nakatsuji 1983) and zebrafish (Bilotta, Barnett et al. 2004).
The chick has been widely used as a model organism for research studies in developmental biology (Eckstein, Shibley et al. 1997; Pourquie 2004; Biau, Bayle et al. 2007). Various aspects of EtOH toxicity during embryonic development have been documented in experimental studies ( $\mathrm{Gu}$, Bailey et al. 2005; Tufan, Abban et al. 2007). Studies carried out on chick embryo address the effects of $\mathrm{EtOH}$ on craniofacial abnormalities (Smith 2008) and angiogenesis (Gu, Bailey et al. 2005). Several studies suggest that EtOH affects the body weight, length and head circumference of chick embryo (Chaudhuri 2004; Kamran, Khan et al. 2011). In addition, it has been shown that microphthalmia may be caused due to EtOH toxicity (Kennelly, Brennan et al. 2011). Some other studies report the negative effects of EtOH on cranial neural crest cells (Smith 1997; Garic-Stankovic, Hernandez et al. 
2005). These studies suggest that EtOH causes severe effects on nervous system, and it also causes the decrease in weight and length of embryo. EtOH has been reported as the leading cause of death in humans (Spies, Sander et al. 2001; Ginter and Simko 2008). Model organism has been used to study the survival rate of EtOH treated embryos (Chaudhuri 2004; Kamran, Khan et al. 2011). The treatment of chick embryo with $\mathrm{EtOH}$ has been reported to decrease the survival rate in chick embryo (Chaudhuri 2004; Kamran, Khan et al. 2011)

Chorioallantoic membrane (CAM) is formed by the fusion of the chorion and allantoic membrane (Ribatti 2008). This membrane is rich vascular network, which serves as respiratory organ of embryo (Ribatti 2008). Chorionallantoic memrane has been widely used for studying the angiogenesis of chick embryo (Ribatti, Gualandris et al. 1997; Ribatti 2008). CAM is formed on day 7 of incubation. EtOH has been reported to affect the process of angiogenesis in CAM assay using chick embryo as model organism (Richardson and Singh 2003; Ribatti 2012). CAM assay is also used in tumor cell invasion and metastasis (Cimpean, Ribatti et al. 2008; Deryugina and Quigley 2008); this is an easy, quick and inexpensive method to study angiogenesis.

Also, yolk sac blood vessels system, which surrounds the early stage embryo, has also been studied to understand angiogenesis (Rosenbruch and Holst 1990; He, Li et al. 2013). In addition, yolk sac blood vessels system is also used to study the cardiovascular system of early chick embryo (He, Li et al. 2013; Karunamuni, Gu et al. 2014). Several studies report an increase in angiogenesis using CAM assay after treatment with EtOH (Gu, Bailey et al. 2005), however, decrease in angiogenesis has been reported on wound healing (Radek, Matthies et al. 2005; Radek, Kovacs et al. 2008) and yolk sac blood vessels system of early staged chick embryos (Karunamuni, Gu et al. 2014). In an elegant study, it was reported that moderate dose of EtOH has positive effects on development of embryo particularly on vasculature of embryo (Gu, Elam et al. 2001). These studies suggest that EtOH might cause an increase or decrease in angiogenesis, depending on EtOH dose and type of blood vessel system.

The chick is the convenient model, which allows us to directly visualize the effects of EtOH on chick development, which enhances our understanding about Fetal Alcoholic Syndrome. Keeping this view in mind, we carried out a study to find out the effects of various concentration of EtOH on survival rate of chick embryo at early stages of development. In addition, we have also set up experiments on early staged chick embryo to assess the effects of various concentrations of EtOH on yolk sac blood vessels, which surround the developing embryo.

\section{Methodology}

Chick eggs were obtained from Poultry Institute of Karachi. These eggs were divided into control and treated groups, for each set of treatment 6 eggs were incubated and the experiment was repeated thrice. Chick eggs were incubated at 37 centigrade in the humidified incubator. After
72 hours of incubation (HH stage 19) these eggs were removed from incubator and the egg shell was rinsed with $70 \%$ EtOH. A small hole was created on the shell of egg and $3 \mathrm{ml}$ of albumin was removed using syringe. Normally developed embryos were used for experiments and all malformed embryos were excluded. The stages of chick embryo development were determined using HamburgerHamilton stages (HH). Chick embryos were treated with $1 \%$, $5 \%, 10 \%, 13 \%$ and $15 \%$ EtOH dissolved in Balanced Salt Solution (BSS) (invitrogen). Control embryos were treated with BSS only. $1 \mathrm{ml}$ of different concentrations of ethanol solutions were poured on the developing embryos, after treating the embryos with a single dose of various concentrations of ethanol solutions, the window of eggs was sealed with transparent tap and the eggs were incubated for 48 hours at 37 centigrade. Preparation of solutions, the dissection procedure and treatments of embryos with various solutions were carried out in biosafety cabinet under aseptic conditions.

The sealed window was open after 48 hours and heart beat of embryos were observed to assess the survival rate. Vessels formation was also observed under microscope and photos were also taken using Digital Camera. Embryos were dissected out and washed with Phosphate buffer saline solution (PBS). Observations were made for any noticeable morphological abnormalities of treated and untreated embryos.

\section{Results}

Treatment with EtOH affected the survival rate and vascular development of embryos in a dose dependent manner. Figure 1 shows the survival rate of embryos. Only slight difference was observed in the survival rate of chick embryo treated with 1 and $5 \%$ of EtOH compared with control (Figure 1), however with increasing dose till $10 \%$ $\mathrm{EtOH}$ the survival rate was reduced to $64 \%$. At $13 \% \mathrm{EtOH}$ survival rate was reduced to half $48 \%$, and at $15 \% \mathrm{EtOH}$ complete death was observed.

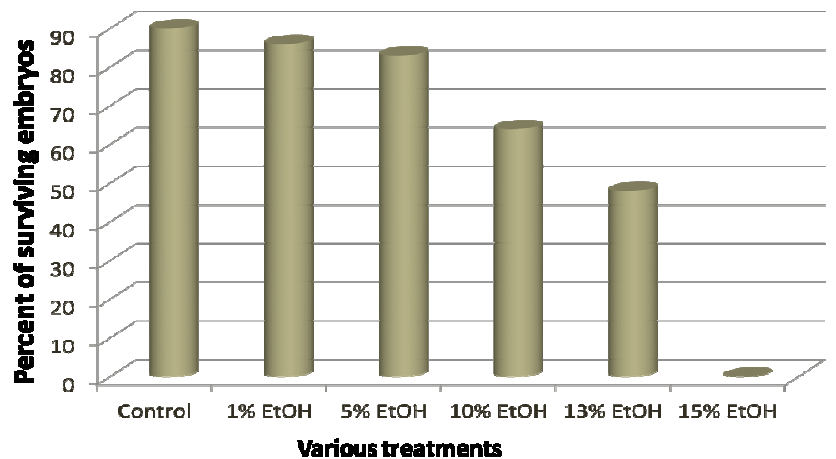

Figure 1. Diagrammatic representation of effects of single dose various concentration of EtOH on survival rate of chick embryos.

EtOH has been reported to negatively and positively affect angiogenesis. In order to find out whether EtOH can affect the angiogenesis we treated the 3 days old chick embryos 
with a single dose of various concentrations of EtOH. Figure 2 shows that treatment of EtOH with $1 \%$ and $5 \%$ did not have any obvious effects on vessels formation (Figure $2 \mathrm{~B}, \mathrm{C}$ ) in comparison with the BSS treated (control) embryos (Figure $2 \mathrm{~A}$ ). However, 10\% EtOH severely inhibited the blood vessels formation (Figure 2 D) and treatment of embryos with $13 \%$ and $15 \%$ EtOH caused severe effects on vessels formation (Figure $2 \mathrm{E}-\mathrm{F}$ ). At $15 \% \mathrm{EtOH}$ vessels were fragmented and embryos turned into the pool of blood (Figure $2 \mathrm{~F}$ ).

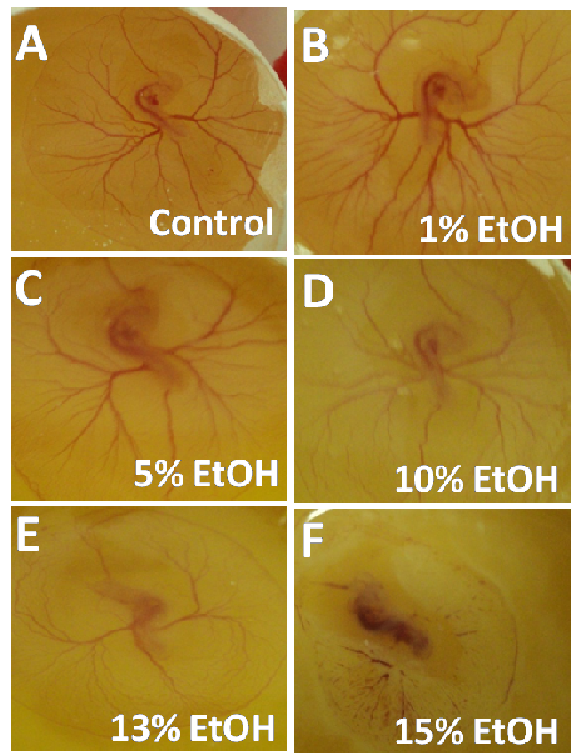

Figure 2. Effects of EtOH on angiogenesis of 5 days old chick embryos.

\section{Discussion}

The data we represent here shows that EtOH affects the survival rate of early stage chick embryos; we have shown that the lethal dose for chick embryo is $15 \%$, our results are not in agreement with the study where it has been shown that $15 \%$ EtOH caused the death of $43 \%$ chick embryo at various stages (Chaudhuri 2004). The difference in finding is due to the fact that we have used a different strategy to treat the embryos with EtOH.

We have found the slight difference in reduction of the length of embryos; these findings are consistent with already published findings but not consistent with the study where they have reported significant difference in weight and height of embryo (Gilani and Persaud 1992; Kamran, Khan et al. 2013). This might be due to the fact that we are studying the early development of chick embryo. Another difference is we have not used the EtOH vapors but we have directly poured various concentrations of EtOH on early stage chick embryos.

EtOH has been reported to increase the angiogenesis using CAM assay (Gu, Bailey et al. 2005; Tay, Heng et al. 2012). The results we represent indicate that moderate dose of EtOH does not affect vessels formation and heart development, which is in agreement with published study (Gu, Elam et al. 2001). However, higher dose at $10 \%$ EtOH inhibit angiogenesis. Inhibition of angiogenesis has also been reported in studies carried out on chick embryo and on wound healing (Radek, Matthies et al. 2005; Radek, Kovacs et al. 2008). This suggests that EtOH inhibit the angiogenesis at higher doses. We have found that at $13 \% \mathrm{EtOH}$ the severe inhibition of the heart is observed. Some other studies have also reported the inhibition of heart at later development of chick embryo (Karunamuni, Gu et al. 2014). This confirms the role of $\mathrm{EtOH}$ in inhibition of heart development. The percentage of $\mathrm{EtOH}>13 \%$ shows marked inhibition of vasculature (Figure $2 \mathrm{E}$ ), these results suggest that EtOH might affect the formation of vessels at early stages of chick development.

\section{Conclusion}

In conclusion, this study demonstrates that the treatment of chick embryo with EtOH at early stages causes decrease in survival rate of chick embryos. This study also suggests that $\mathrm{EtOH}$ is harmful for blood vessels, which supply embryo with nutrients at the early stages of development. Collectively, the date provides an insight into the understanding of effects produced by $\mathrm{EtOH}$.

\section{References}

[1] Biau, S., S. Bayle, et al. (2007). "The chick embryo: an animal model for detection of the effects of hormonal compounds." Anal Bioanal Chem 387(4): 1397-403.

[2] Bilotta, J., J. A. Barnett, et al. (2004). "Ethanol exposure alters zebrafish development: a novel model of fetal alcohol syndrome." Neurotoxicol Teratol 26(6): 737-43.

[3] Chaudhuri, J. D. (2004). "Effect of a single dose of ethanol on developing skeletal muscle of chick embryos." Alcohol 34(23): 279-83.

[4] Cimpean, A. M., D. Ribatti, et al. (2008). "The chick embryo chorioallantoic membrane as a model to study tumor metastasis." Angiogenesis 11(4): 311-9.

[5] Deryugina, E. I. and J. P. Quigley (2008). "Chapter 2. Chick embryo chorioallantoic membrane models to quantify angiogenesis induced by inflammatory and tumor cells or purified effector molecules." Methods Enzymol 444: 21-41.

[6] Eckstein, L. W., I. A. Shibley, Jr., et al. (1997). "Changes in brain glucose levels and glucose transporter protein isoforms in alcohol- or nicotine-treated chick embryos." Brain Res Dev Brain Res 103(1): 59-65.

[7] Garic-Stankovic, A., M. R. Hernandez, et al. (2005). "Ethanol triggers neural crest apoptosis through the selective activation of a pertussis toxin-sensitive $G$ protein and a phospholipase Cbeta-dependent Ca2+ transient." Alcohol Clin Exp Res 29(7): $1237-46$

[8] Ginter, E. and V. Simko (2008). "Ethanol and cardiovascular diseases: epidemiological, biochemical and clinical aspects." Bratisl Lek Listy 109(12): 590-4.

[9] Gu, J. W., A. P. Bailey, et al. (2005). "Ethanol stimulates tumor progression and expression of vascular endothelial growth factor in chick embryos." Cancer 103(2): 422-31. 
[10] Gu, J. W., J. Elam, et al. (2001). "Moderate levels of ethanol induce expression of vascular endothelial growth factor and stimulate angiogenesis." Am J Physiol Regul Integr Comp Physiol 281(1): R365-72

[11] He, R. R., Y. Li, et al. (2013). "A new oxidative stress model, 2,2-azobis(2-amidinopropane) dihydrochloride induces cardiovascular damages in chicken embryo." PLoS One 8(3): e57732.

[12] Kamran, K., M. Y. Khan, et al. (2011). "Teratogenic effects of ethanol vapour exposure on chick embryos." J Pak Med Assoc 61(4): 328-31.

[13] Kamran, K., M. Y. Khan, et al. (2013). "Ethanol vapour induced dilated cardiomyopathy in chick embryos." J Pak Med Assoc 63(9): 1084-8.

[14] Karunamuni, G., S. Gu, et al. (2014). "Ethanol exposure alters early cardiac function in the looping heart: a mechanism for congenital heart defects?" Am J Physiol Heart Circ Physiol 306(3): H414-21.

[15] Kennelly, K., D. Brennan, et al. (2011). "Histological characterisation of the ethanol-induced microphthalmia phenotype in a chick embryo model system." Reprod Toxicol 32(2): 227-34.

[16] Lange, S., K. Shield, et al. (2013). "Prevalence of fetal alcohol spectrum disorders in child care settings: a meta-analysis." Pediatrics 132(4): e980-95.

[17] Nakatsuji, N. (1983). "Craniofacial malformation in Xenopus laevis tadpoles caused by the exposure of early embryos to ethanol." Teratology 28(2): 299-305.

[18] Nakatsuji, N. and K. E. Johnson (1984). "Effects of ethanol on the primitive streak stage mouse embryo." Teratology 29(3): 369-75.

[19] Popova, S., S. Lange, et al. (2011). "Fetal alcohol spectrum disorder prevalence estimates in correctional systems: a systematic literature review." Can J Public Health 102(5): 336-40.

[20] Pourquie, O. (2004). "The chick embryo: a leading model in somitogenesis studies." Mech Dev 121(9): 1069-79.
[21] Radek, K. A., E. J. Kovacs, et al. (2008). "Acute ethanol exposure disrupts VEGF receptor cell signaling in endothelial cells." Am J Physiol Heart Circ Physiol 295(1): H174-84.

[22] Radek, K. A., A. M. Matthies, et al. (2005). "Acute ethanol exposure impairs angiogenesis and the proliferative phase of wound healing." Am J Physiol Heart Circ Physiol 289(3): H1084-90.

[23] Ribatti, D. (2008). "Chick embryo chorioallantoic membrane as a useful tool to study angiogenesis." Int Rev Cell Mol Biol 270: 181-224.

[24] Ribatti, D. (2012). "Chicken chorioallantoic membrane angiogenesis model." Methods Mol Biol 843: 47-57.

[25] Ribatti, D., A. Gualandris, et al. (1997). "New model for the study of angiogenesis and antiangiogenesis in the chick embryo chorioallantoic membrane: the gelatin sponge/chorioallantoic membrane assay." J Vasc Res 34(6): 455-63.

[26] Richardson, M. and G. Singh (2003). "Observations on the use of the avian chorioallantoic membrane (CAM) model in investigations into angiogenesis." Curr Drug Targets Cardiovasc Haematol Disord 3(2): 155-85.

[27] Rosenbruch, M. and A. Holst (1990). "The chick embryo yolk-sac blood vessel system as an experimental model for irritation and inflammation." Toxicol In Vitro 4(4-5): 327-31.

[28] Smith, S. M. (1997). "Alcohol-induced cell death in the embryo." Alcohol Health Res World 21(4): 287-97.

[29] Smith, S. M. (2008). "The avian embryo in fetal alcohol research." Methods Mol Biol 447: 75-84.

[30] Spies, C. D., M. Sander, et al. (2001). "Effects of alcohol on the heart." Curr Opin Crit Care 7(5): 337-43.

[31] Tay, S. L., P. W. Heng, et al. (2012). "The chick chorioallantoic membrane imaging method as a platform to evaluate vasoactivity and assess irritancy of compounds." J Pharm Pharmacol 64(8): 1128-37.

[32] Tufan, A. C., G. Abban, et al. (2007). "The effect of in ovo ethanol exposure on retina and optic nerve in a chick embryo model system." Reprod Toxicol 23(1): 75-82. 